\title{
Authentication of data hiding using co-occurrence matrix
}

Melad jader saeed

\section{meladjader@uomosul.edu.iq Ghadathanoon@uomosul.edu.iq}

University of Mosul/ College of Computer Science and Mathematics

Received on : 28/3/2011

\section{Ghada thanoon}

Accepted on :21/6/2011

\begin{abstract}
This research is considered one of the steps aiming to deal with one of the most important challenges facing communicators via Internet, as a means for ensuring information security and verifying the authenticity and credibility of the received message. The research designs and implements proposed system for verifying the authenticity of retrieved information which are ciphered and hidden inside colored images. This is done through utilizing the capabilities and features that the process of image texture analysis offers, using the co-occurrence matrix. The message authenticity is verified by comparing the co-occurrence matrices before sending and after receiving in order to assure non infiltration.

Results of applying this system on image files with different extensions, and on text messages with different sizes too, have shown supremacy in fighting spam. Keyword: data hiding, authentiacation, co-occurrence,cipher.

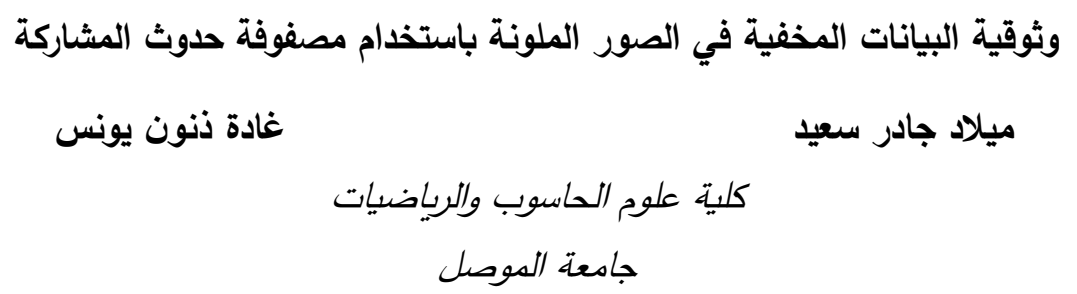

تاريخ القبول: 2011/6/21

تاريخ الاستلام: 2011/3/28
\end{abstract}

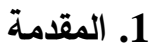

من المعايير الأساسية لأمن البيانات والتي من الواجب أن تتوفر في نظم المعلومات هي الوثوقية

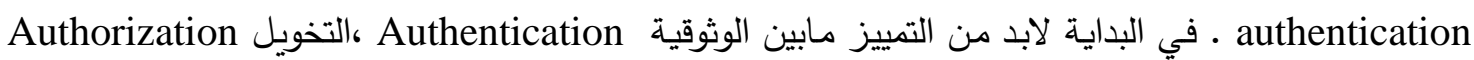


والتحكم في النفاذ Access Control يقصد بالوثوقية: الإجرائية التي يقر النظام بمقتضـاها وصول المستخدم إلى

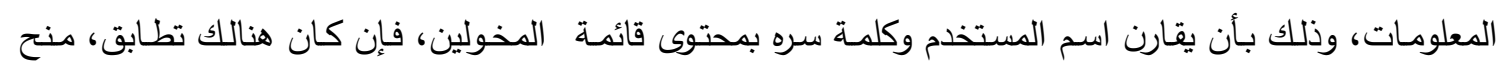

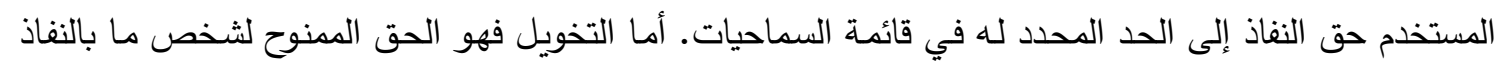

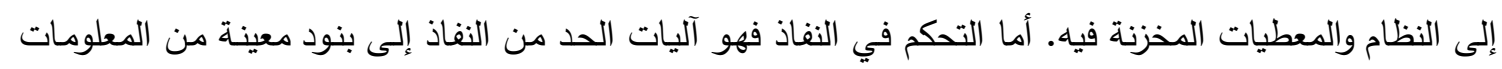

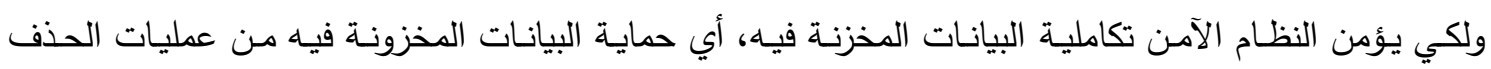

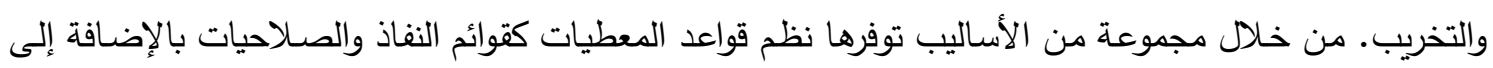
علاقات الترابط Referential Integrity مابين البيانات المخزنة فيه. كما يؤمن النظام الآمن تكامل البيانات المرسلة لهعرفة فيما إذا تم تعديل أو حذف أي جزء منها أو أنها غير مكررة، وتحقيق ذلك يمكن أن يتم من خلال

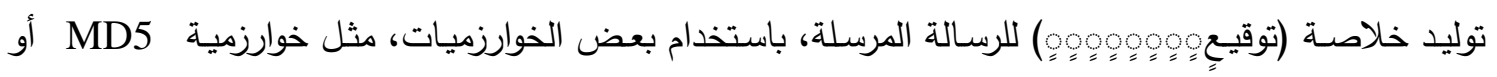
خوارزمية SHA، وتضمين هذه الخلاصة مع كل رسالة ترسل عبر الثبكة، وبالتالي التأكد من أن الرسالة صحيحة

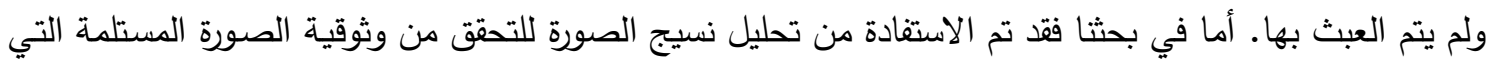

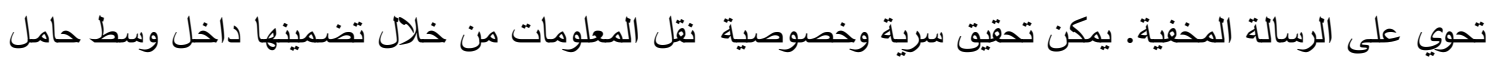
لها وهناك عدة اتفاقيات وتقنيات تضمين تمكننا من إخفاء المعلومات في شيء معين وجميع الاتفاقيات والتقنيات يجب أن تحقق عددا من المنطلبات لكي يمكن تطبيق نظرية إخفاء المعلومات بصورة صحيحة, وفي أدناه مجموعه من المتطلبات الرئيسية. 11]

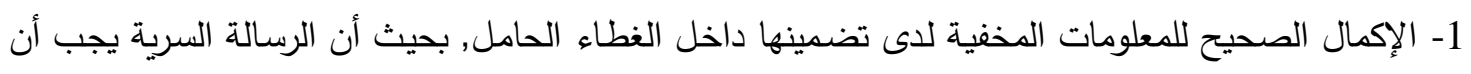
لا تتغير إذ أن تغيير البيانات المضمنة يعني فشل العملية. 2- الوسط الناقل الذي يغطي الرسالة السرية يجب أن لا يتغير أيضـا وعلى الأقل أن لا تكون تغييراته ظاهرة

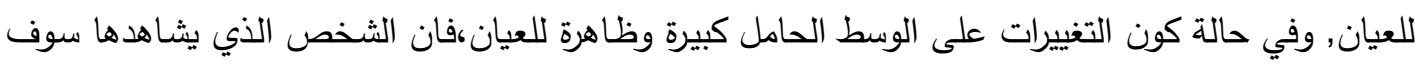
يعلم بوجود معلومات مخفيه داخله فيحاول أن يفتحها أو يدمرها. 3- يؤخذ بنظر الاعتبار دائما أن المهاجم على علم بوجود معلومات مخفية داخل فات الغطاء الحامل.

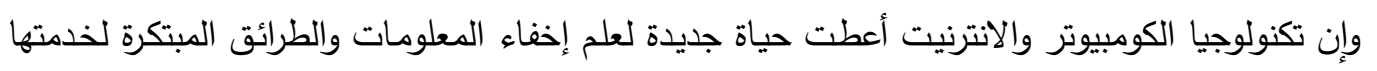

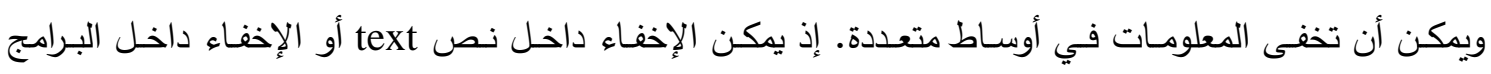
software أو الإخفاء في مساحة القرص أAisk space والإخفاء في الصوت Audio أو الإخفاء في الصور .Image

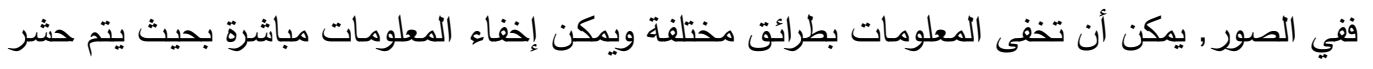

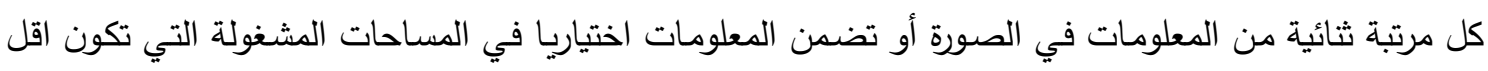
إدراكا. وقد تتبعثر المعلومات بصورة عشوائية أو تتكرر بضع مرات في كل مكان من الصورة. ويمكن الإخفاء في

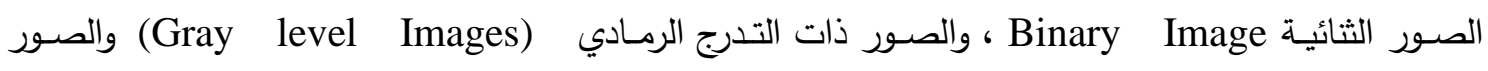
الملونة (Color Images): والتي تم اعتمادها في هذا البحث إذ انه منذ ظهور الصور الملونة ومهع مرور الزمن

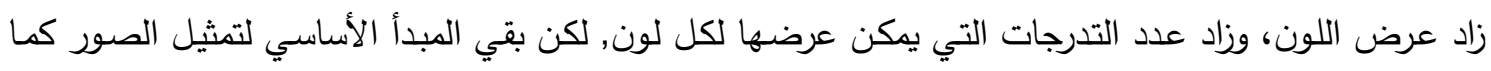

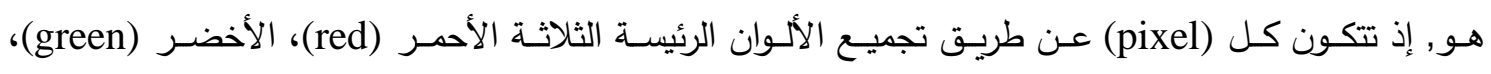

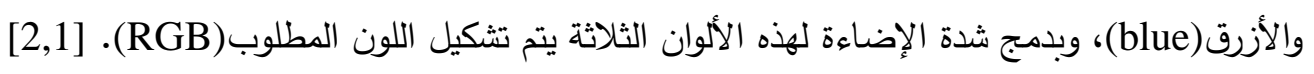


2- طرق الإخفاء :

هناك عدد كبير من طرائق الإخفاء في الصور من أشهرها: [3]

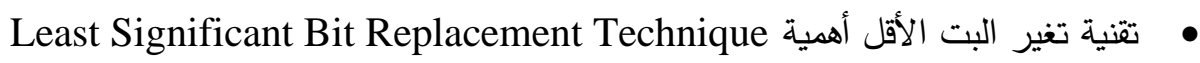

Moderate Significant Bit Replacement Technique تقنية تغير البت الوسطي •

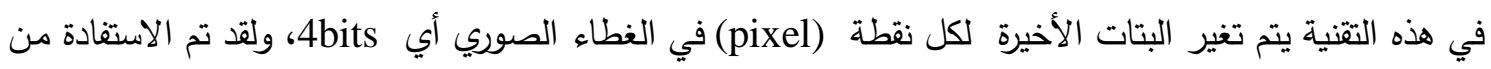

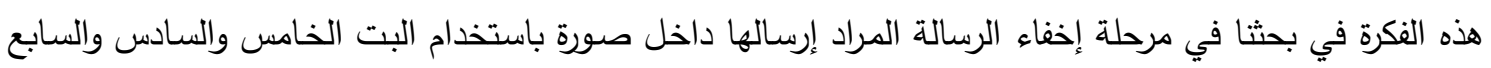

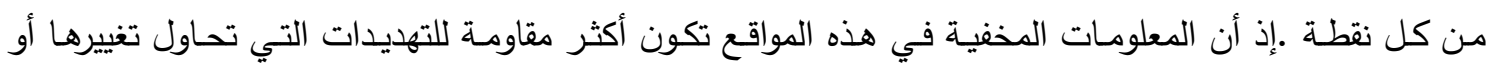
إزالتها. وكما هو معلوم إن هذا التغيير يؤدي إلى ظهور تشوهات في الصورة ولقد تم تجاوز هذه النقطة بتتفيذ

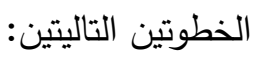
• القيام بعملية إخفاء البيانات في إطار الصورة فقط وقد تم البدء من التدرج الأحمر ثم الأخضر ثم الأزرق. • • • • • •

ومن خلال تطبيق النقطتين السابقتين تم إنتاج صورة لا تحوي أي تشوه ممكن أن يثير الثك للمتطفل.

3- - 2 - الأعمال السابقة:

من خلال قراءة عدد من البحوث تم استتباط أفكار عدة ومن ثم دراستها وتطوير البعض منها للوصول

إلى النتائج الموضحة، ففي البحث [3] تم إخفاء نص داخل صورة ملونـة باستخدام طريقة الإخفاء بتغير البتات

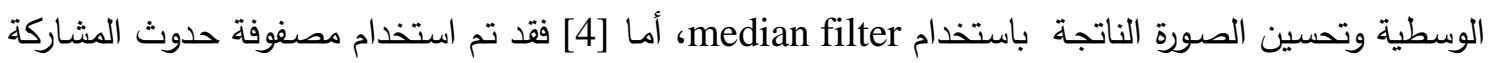

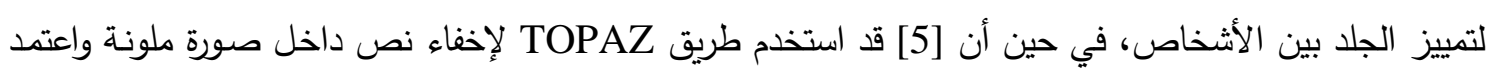

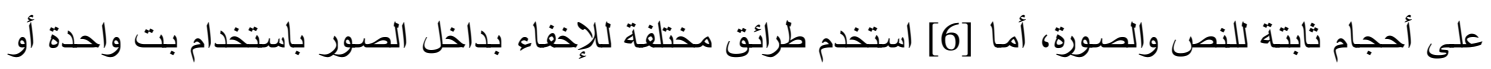
اثثين أو ثلاثة أو أربعة ودراسة مميزات كل طريقة.

4- تحويل فورير السريع Fast Fourier Transformation:

هو احد أنواع التحويلات (Transformation) التي تتقل الصورة من صيغة إلى أخرى ولكي تعالج

الصورة أو الإثارة على الحاسوب يجب أن يتم تحويلها إلى القيم غير المستمرة (Discrete) أي ثابتة أو متقطعة ،

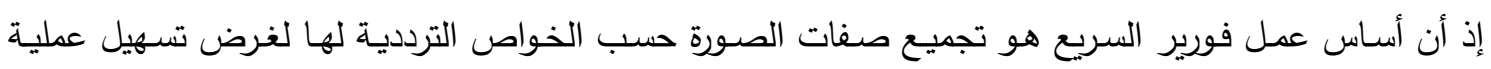
المعالجة، ويتم حسابه حسب المعادلة الآتية [2]:

$F(u)=\int_{-\infty}^{\infty} F(x) e^{-j 2 \prod u x}$

5igital Image Enhancement تحسين الصور الرقمية

يعرف التباين بأنه تدرج و توزيع قيم وحدات الصورة الرقمية على المقياس من 0 إلى 255 المستخدم بواسطة الحاسوب

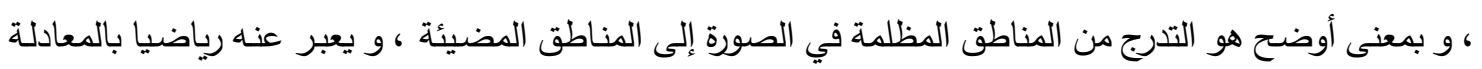

$\mathrm{C}=(\operatorname{Imax}-\operatorname{Imin}) /(\operatorname{Imax}+\operatorname{Imin})$

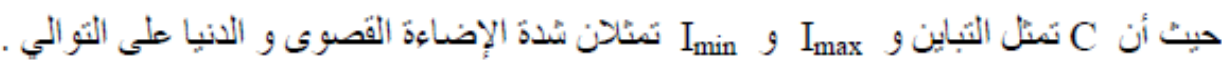


وحتى يسهل تفسير الصورة يتم تحسينها إما بتغيير التباين ليشمل التدرج الرمادي أو تحويل التدرج الرمادي إلى تدرج لوني [7, 2].

و لإجراء تحسين لهذا التباين أو للوضوح الإشعاعي للصورة هنالك تقنيات متعددة و جل هذه التقنيات تتطلق من مبدأ تمديد التدرج الرمادي أو توزيع الأعداد الرقمية لوحدات الصورة بحيث تغطي كل المدى المدكن، أي أي من السواد

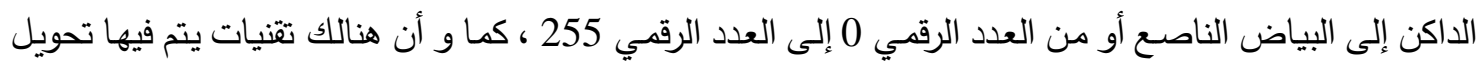
التدرج الرمادي في الصورة إلى ألوان زائفة، كل ذلك الغرض منده تسهيل عملية تفسير الصورة واستتباط المعلومات.

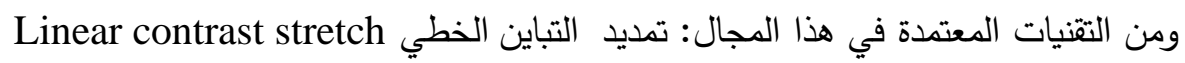

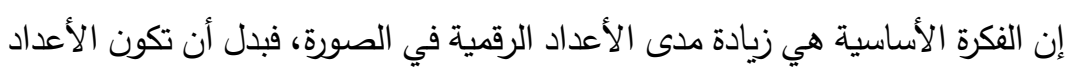

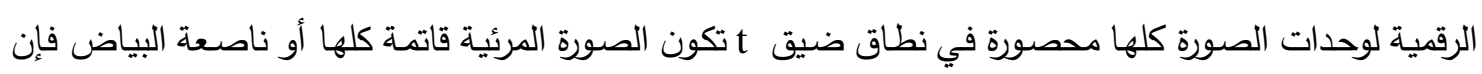
الهدف هو توزيع الأعداد الرقمية للصورة لتشمل جميع المدى المتاح وهو من 0 إلى 255 ، حتى يكون هنالك مدى لـى تباين واسع بين وحدات الصورة و يسهل من عملية تفسير الصورة المرئية.

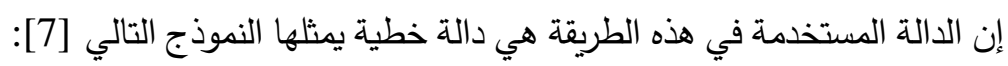
$\mathrm{DNo}=255[(\mathrm{DNi}-\mathrm{DNmin}) /(\mathrm{DNmax}-\mathrm{DNmin})]$

$$
\begin{aligned}
& \text { حيث أن DN = العدد الرقمي المخرج لوحدة صورة } \\
& \text { = العدد الرقمي، الأصلي (المدخل) لوحدة الصنورة } \\
& \text { = أقلّل عدد رقمبي في البيانات المخلةً } \\
& \text { = أقصى عدد رقمب في البيانات المدخلة }
\end{aligned}
$$

ولأنها تستخدم على صور أحادية ولما يتطلبه العمل البحثي فقد تم تطبيق هذه المعادلة على جزء الأحمر

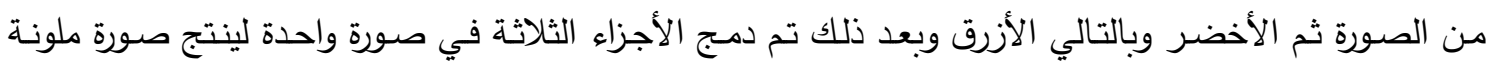
وبالتالي تم تحسين الصورة الملونة باستخدام هذه الطريقة التي أثتتت أنها لا تسبب فقداناً بالمعلومات أثناء إداء إجراء عملية التحسين. [7, 2]

6- خواص مستندة إلى النسجة Texture Based Features:

إن النسجة (texture) واحدة من أكثر الخواص التي تستخدم لتحليل الصور وتفسيرها، فالنسجة هي مقياس لاختلاف كثافة السطح، وتحدد بعض خواص الصـورة، وقد طبق العديد من الأسـاليب لتحليل النسجة

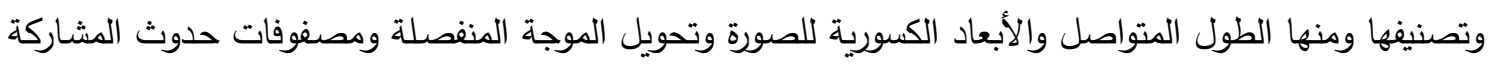

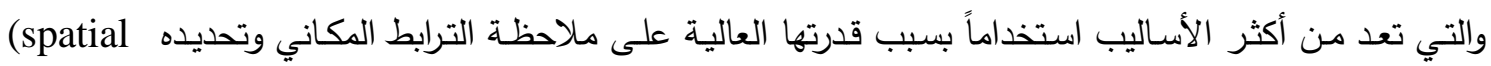
dependence)

\section{1-6 مصفوفة حدوث المشاركة Co-occurrence matrix:}

إن هذه المصـوفات اقترحهـا العـالم Haralick في عـام 1973، وهي مصـفوفة ثنائيـة الأبعـاد (معتمـدة على تصى المستويات الرمادية)، تستخدم بثكل أساسي في تحليل النسجة بسبب قدرتها العالية على تحديد الترابط المكاني لقيم 
المستويات الرمادية في الصورة، إذ تعمل مصفوفة حدوث المشاركة (P) بوصفها جامعاً تراكميا (accumulator)

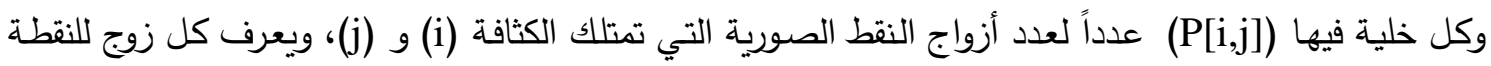
الصورية بالبعد والاتجاه الذي يمكن أن يمثل بمتجه الإزاحة (dx,dy)(dx) )، و (dx) تمثل إزاحة النقطة الصورية

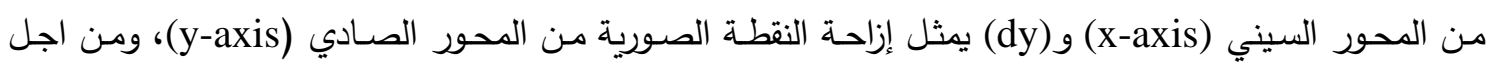
تحديد الاعتماد المكاني لقيم المستوى المكاني تم حساب عدد من خواص النسجة ، المذكورة في الجدول (1) والتي اعتمدت للتأكد من وثوقية الصورة المرسلة [4]، وهي:

جدول (1). خواص النسجة

\begin{tabular}{|c|c|c|}
\hline الصيغة العامة & الصفة & $ت$ \\
\hline$\sum_{i}^{M} \sum_{j}^{N} \mathrm{P}[i, j] \log \mathrm{P}[i, j]$ & Entropy العشوائية & 1 \\
\hline$\sum_{i}^{M} \sum_{j}^{N} \mathrm{P}^{2}[i, j]$ & Energy الطاقة & 2 \\
\hline$\sum_{i}^{M} \sum_{j}^{N}(i-j)^{2} \mathrm{P}[i, j]$ & Contrast التباين & 3 \\
\hline$\sum_{i}^{M} \sum_{j}^{N} \frac{\mathrm{P} \mid[i, j]}{1+|i-j|}$ & Homogeneity التجانس & 4 \\
\hline$\frac{1}{2} \sum_{i}^{M} \sum_{j}^{N}(i p[i, j]+j p[i, j])$ & المتوسط Mean & 5 \\
\hline$\frac{1}{2} \sum_{i}^{M} \sum_{j}^{N}\left((i-\mu)^{2} p[i, j]+(j-\mu)^{2} p[i, j]\right)$ & الاختلاف Variance & 6 \\
\hline$\sum_{i}^{M} \sum_{j}^{N} \frac{(i-\mu)(j-\mu) p[i, j]}{\sigma^{2}}$ & Correlation الارتباط & 7 \\
\hline$\sum_{i}^{M} \sum_{j}^{N} \frac{p[i, j]}{|i-j|^{k}} i \neq j$ & عزم الاختلاف المعكوس & 8 \\
\hline
\end{tabular}

7- التكميم الخطي والتشفير Quantize \& encode:

تتم عملية تكميم خطي وتثفير للبيانات الحقيقية الددخلة من نوع floating point وإخراجها على شكل بيانات من نوع integer ـ فكرة هذه الطريقة تعتمد على تكميم المصفوفة المدخلة التي تحوي على بيانات حقيقية وتثفيره كبيانات من نوع integer باستخدام

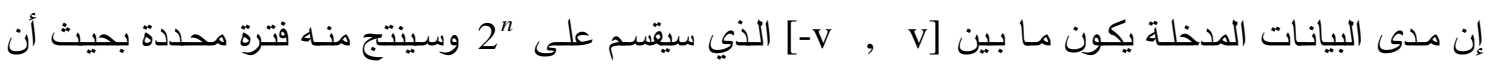
المخرجات الناتجة من عملية التكميم الخطي يجب أن تقع ضمن أول فترة حصلنا عليها من عملية التقسيم وتستمر

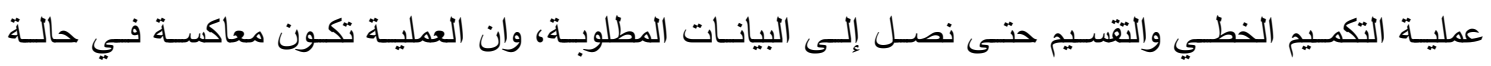
[8].dequantize encode 8- الخوارزمية المقترحة في الإرسال: 
Bmp, Jpg, Gif المدخلات: النص المطلوب إرساله للطرف الآخر، صورة ملونة بامتداد 1. البداية.

2. تجزئة الصورة إلى المكونات الأساسية (الأحمر والأخضر والأزرق) . 3. تحويل النص إلى نظام الاسكي ومن ثم الثنائي. 4. إخفاء النص بإطار الجزء (الأحمر ) من الصورة ابتداءً من الأعلى ثم الأسفل ثم الأيسر ثم الأيمن وباستخدام طريقة (تغير البتات الأخيرة) ولقد تم استخدام البت 5 و6 و و 7.

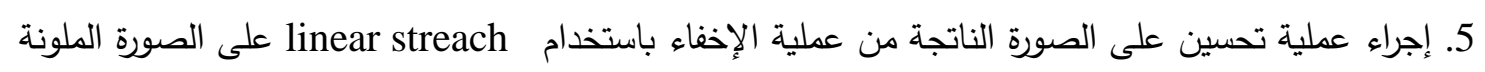
من اجل القضاء على أي تشوه يحدث على الصورة جراء عملية الإخفاء. 6. إجراء عملية طرح بين الصورة التي تحوي الإخفاء و الصورة بعد التحسين لينتج صورة جدئ الصيدة. 7. إجراء عملية ترميز على الصورة الجديدة باستخدام Quantization encoding لينتج الصورة المرمزة.

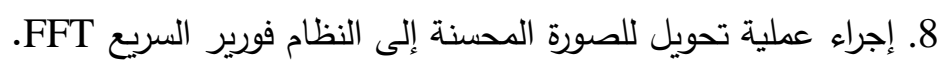
9. دمج نقاط الصورة المرمزة مع الصورة المحولة إلى FFT وإنتاج صورة مبهمة المعاد المعالم. 10. حساب مصفوفة حدوث المشاركة للصورة الناتجة. 11. تجزئة الصورة إلى المكونات الأساسية (الأحمر والأخضر والأزرق).

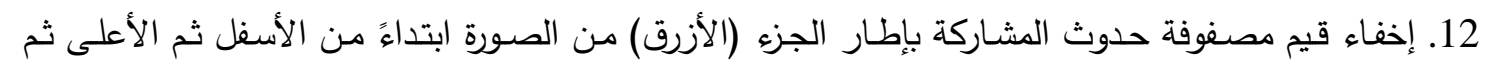
الأيسر ثم الأيمن و باستخدام طريقة (تغير البت الوسطي). 13. ومن ثم إرسال الصورة الأخيرة. 14. النهاية. الثكل (1) يوضح المخطط الصندوقي للخوارزمية المقترحة. 9- الخوارزمية المقترحة في الاستلام:

المدخلات: الصورة المدموجة بعد إخفاء مصفوفة حدوث المشاركة فيها. 1. البداية.

2. استلام الصورة المدموجة والحاوية على مصفوفة حدوث المشاركة التي سوف تستخدم للتحقق من الوثوقية.

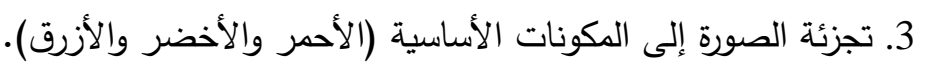

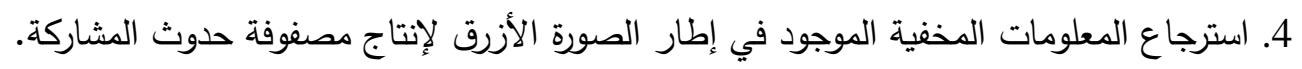
5. حساب مصفوفة حدوث المشاركة للصورة الناتجة بعد استرجاع المعلومات المخفية.

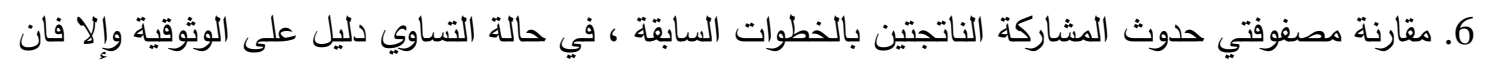

$$
\text { الصورة محرفة وسوف تتوقف الخوارزمية. }
$$

7. فك دمج الصورتين عن طريق توزيع النقاط الفردية لتمثل الصورة المحولة إلى FFT ، والنقاط الزوجية لتمثل

$$
\text { الصورة المرمزة. }
$$

8. تحويل الصورة المحولة إلى النظام المكاني Spatial Domain باستخدام transform

9. فك رمز الصورة المرمزة باستخدام Dequantization decoding. 
10. جمع الصورة المحسنة مع الصورة بعد فك الترميز منها لتتتج الصورة التي تحوي الإخفاء. 11. تجزئة الصورة إلى المكونات الأساسية (الأحمر والأخضر والأزرق).

12. استرجاع المعلومات المخفية الموجودة في إطار الصورة الأحمر لينتج الرسالة المراد إرسالها. 13. النهاية.

المخرجات: الرسالة، الصورة الحاملة للرسالة. الثكل (2) يوضح المخطط للخوارزمية المقترحة للاستلام.

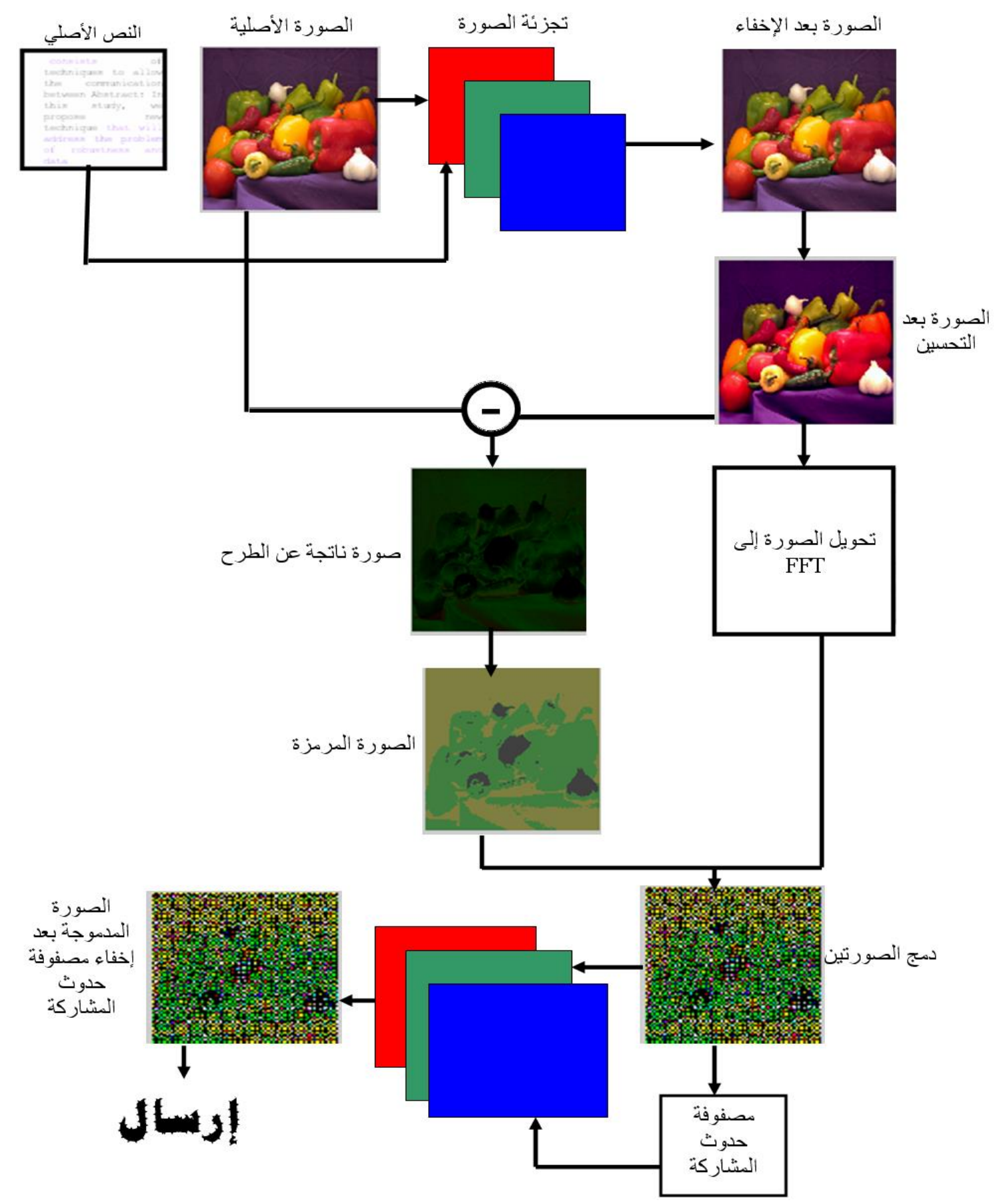

شكل (1). مخطط يمثل الخوارزمية المقترحة للإرسال 


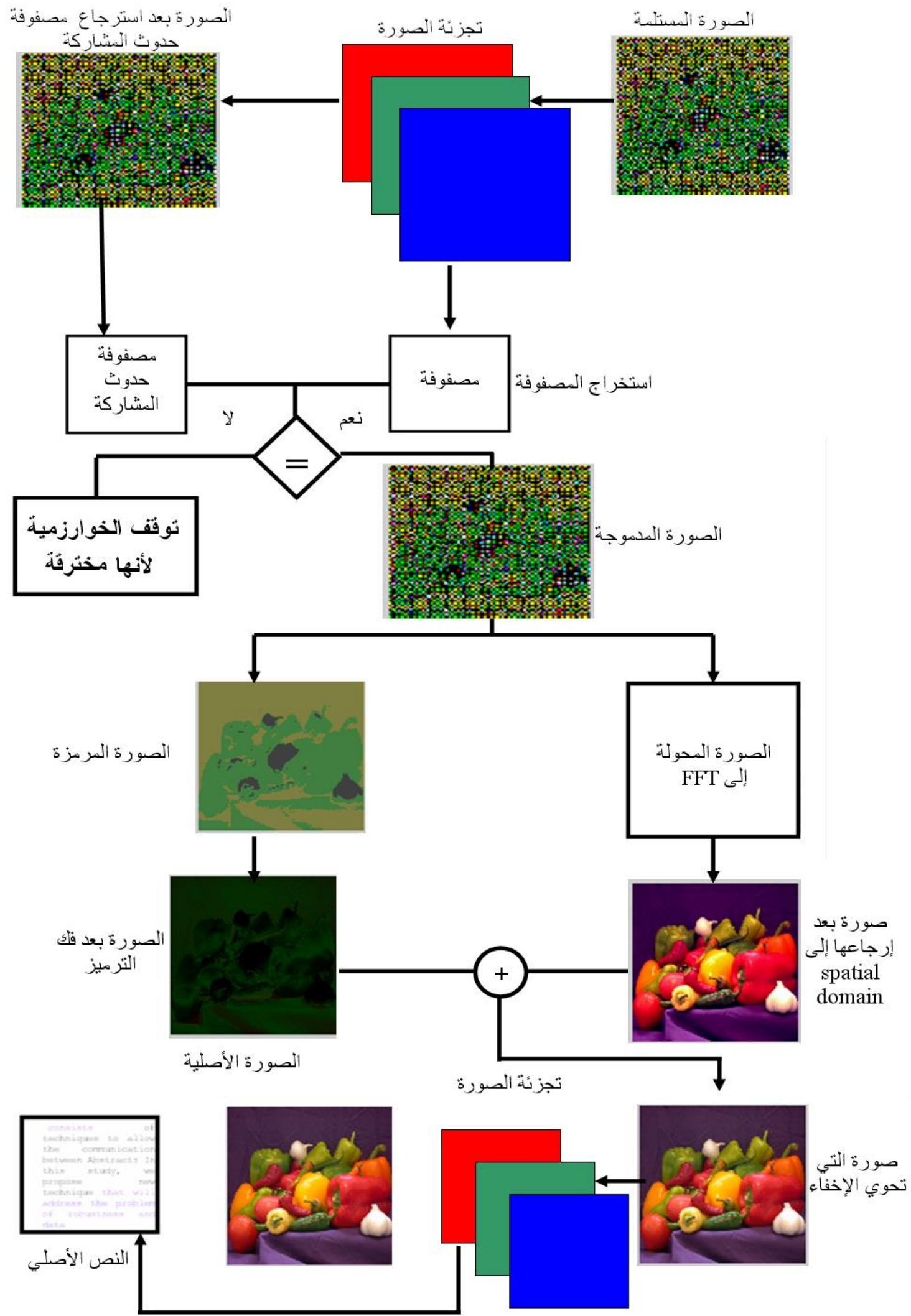

شكل (2). مخطط يمثل الخوارزمية المقترحة للاستلام 
10 - 10 مناقشة النتائج:

من خلال تطبيق الخوارزمية على أكثر من صورة تختلف بالنوعية والامتداد أثبتت الخوارزمية نجاحها من خلال

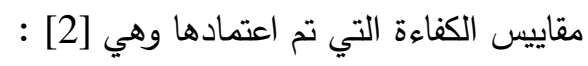
1. مقياس مقدار التشوه: وهو قيمة الكفاءة المستخدمة لقياس مدى التشوه في الصورة الناتجة من عملية الإخفاء

$$
\begin{aligned}
& \mathrm{PSNR}=10 \log _{10}\left(\frac{C_{\max }^{2}}{M S E}\right) \\
& \mathrm{MSE}=\frac{1}{M N} \sum_{x=1}^{M} \sum_{y=1}^{N}\left(S_{x y}-C_{x y}\right)^{2}
\end{aligned}
$$$$
\text { باعتماد المعادلة الآتية: }
$$

$$
\text { : أعلى قيمة لونية في الصورة. }
$$

$$
\begin{aligned}
& \text { M, N } \\
& \text { S : تمثل الصورة الأصلية (الغطاء). } \\
& \text { : تمثل الصورة التي تحوي المعلومات المخفية. }
\end{aligned}
$$

2. - معامل الارتباط Correlation Function:

تستخدم هذه الدالة لغرض إجراء مقارنـة بين مصفوفتين وملاحظة مدى التقارب بينهما فكلما كانت القيمـة الناتجة قريبة من الواحد كان ذلك دليلاً على درجة الوضوحية وقلة وجود التثوه [2].

$$
\text { القانون العام لها }
$$

$\mathrm{A}=\operatorname{corr} 2(\mathrm{p} 1, \mathrm{p} 2)$

P1: الصورة الأصلية (الغطاء).

P2: الصورة التي تحوي المعلومات المخفية.

- فيما يلي بعض الأمثلة العملية التي توضعح خطوات الخوارزمية المقترحة وقيم المقاييس المستخدمة، وفي

جميع الحالات تم إرجاع النص الأصلي بدون تحريف وكان النص الأصلي: توضئ

consists of techniques to allow the communication
between Abstract: In this study, we propose new
technique that will address the problem of
robustness and data

ويمكن تحديد حجم النص الذي ممكن إخفاؤه داخل الصورة المستخدمة كغطاء من خلال المعادلة التالية: No. of letter in message $=($ size of image $* 11 * 3) / 8$

وذلك لأنه الإخفاء يكون في إطار الصورة الأحمر في الأعلى ثم الأسفل ثم الأطراف يليه إطار الصورة

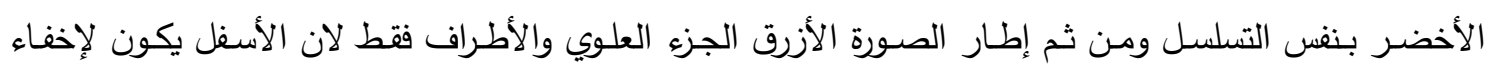

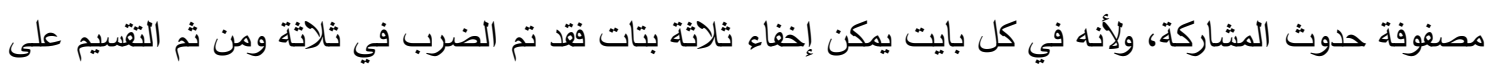

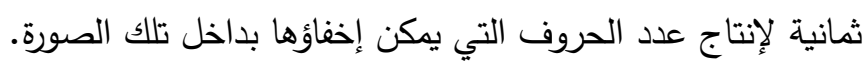




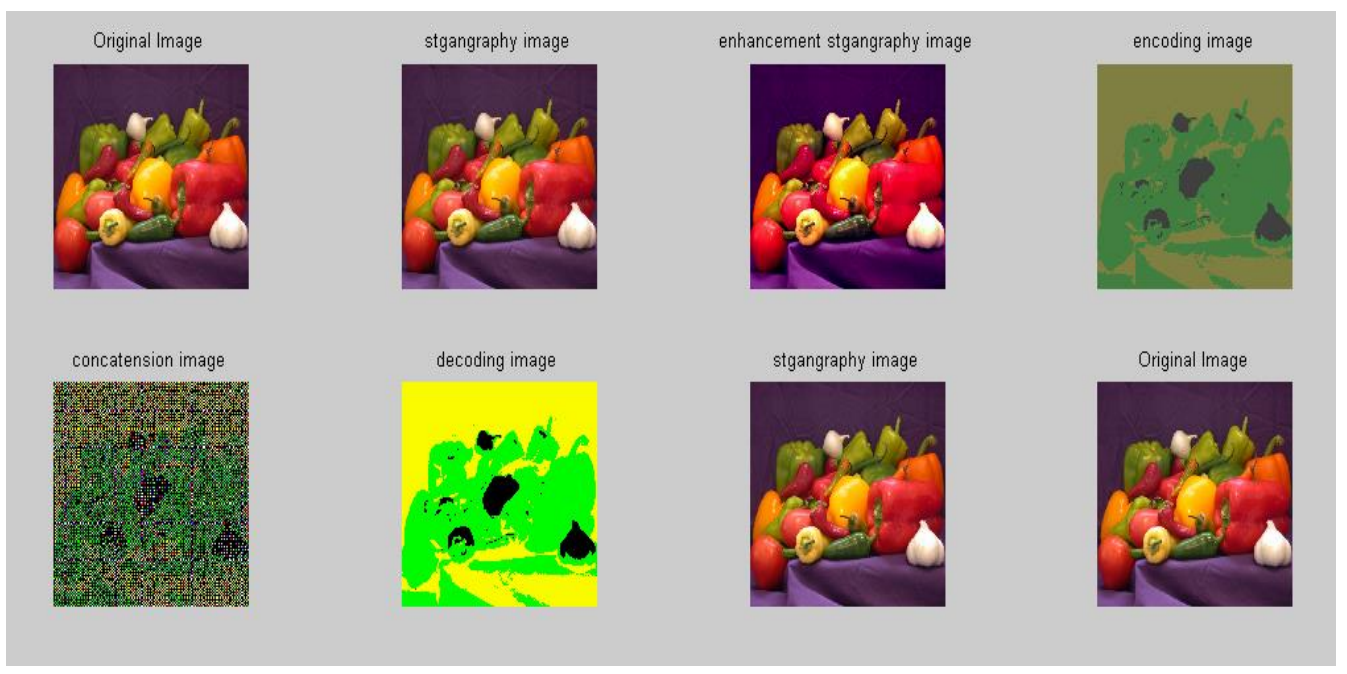

شكل (3). صورة الفواكه

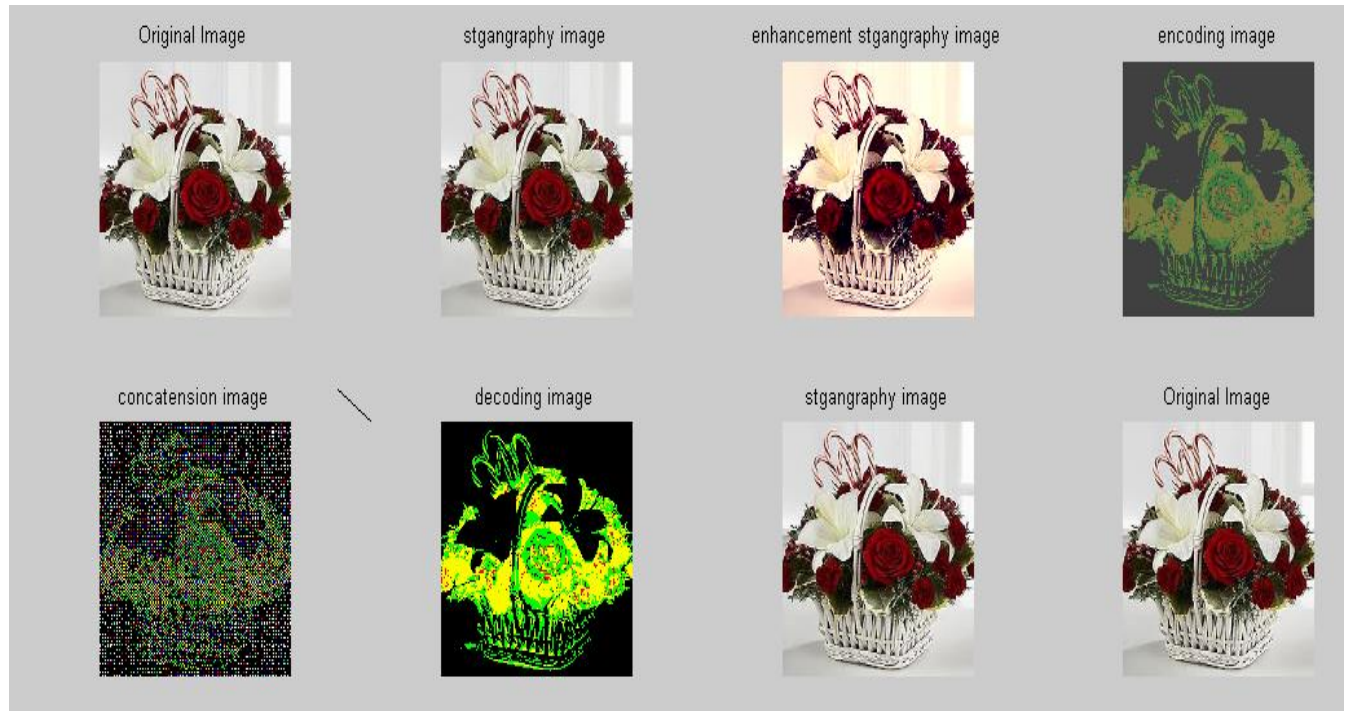

شكل (4). صورة سلة الورد

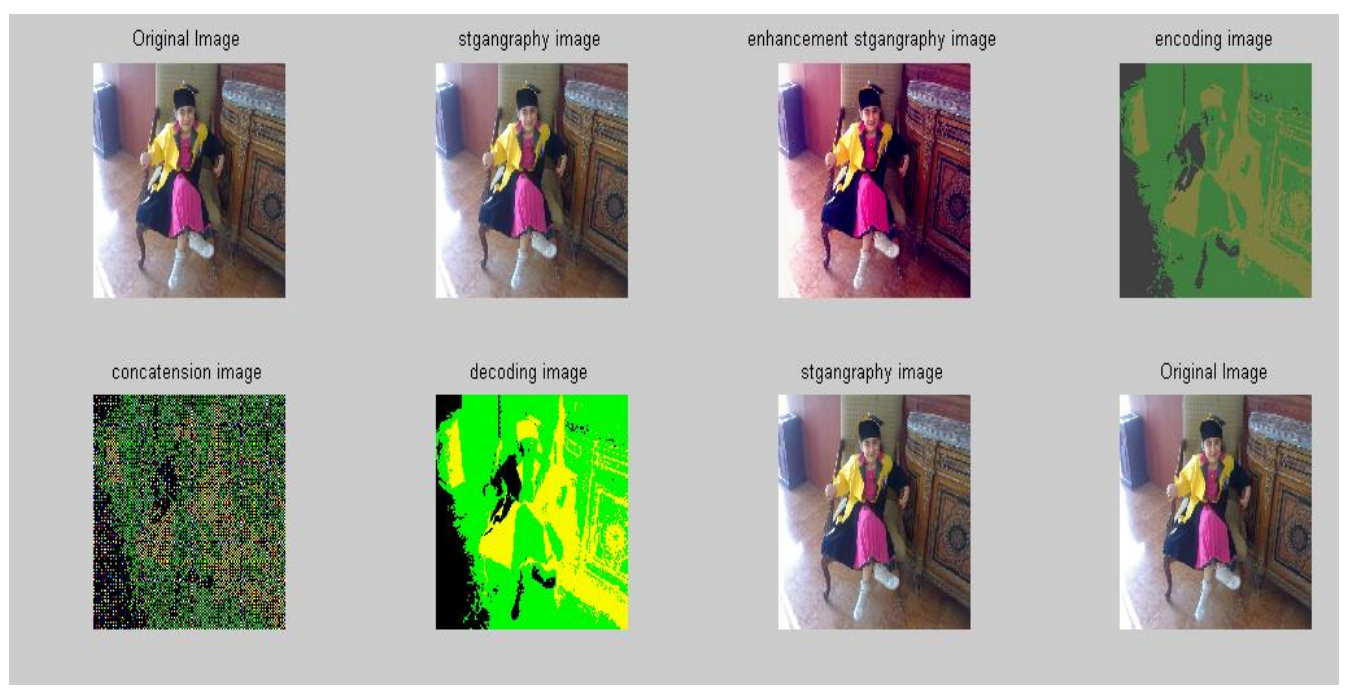

شكل (5). صورة البنت 


$$
\text { جدول (2). مقاييس كفاءة }
$$

\begin{tabular}{|c|c|c|c|c|}
\hline نسبة استرجاع النص & قيمة Correlation & قيمة PSNR & نوعها & اسم الصورة \\
\hline$\% 100$ & 1 & $65.7340 \mathrm{db}$ & JPG & صورة الفواكه \\
\hline$\% 100$ & 1 & $59.6248 \mathrm{db}$ & JPEG & صورة سلة الورد \\
\hline$\% 100$ & 1 & $58.6763 \mathrm{db}$ & BMP & صورة البنت \\
\hline
\end{tabular}

أثتتت النتائج العملية كفاءة الخوارزمية المقترحة من ناحية الوثوقية وان المعلومات المخفية لم يحدث لها أي تغير أو تشوه على الملف الصوري الذي يعد غطاء ، إذ تم قياس مدى صلاحية ووضوحية الصورة الناتجة بعد

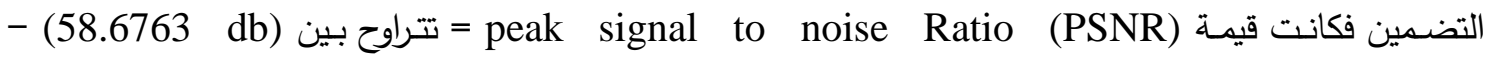
Correlation كانت مساوية للا (1) في (65.0734db) جميع الحالات.

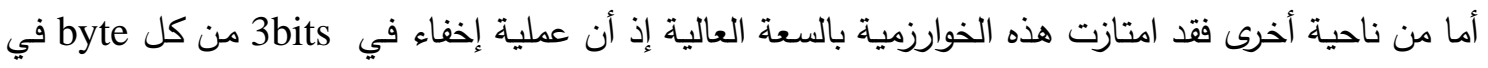

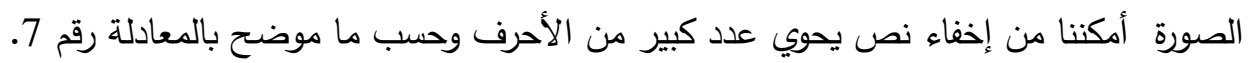

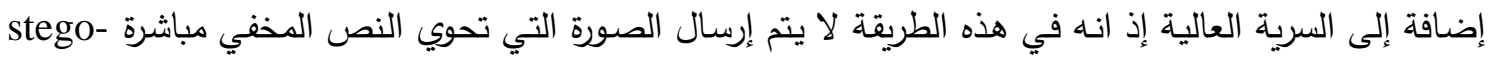
imag ولكن يمر بعدد من المراحل للتغير ومن ثم إرساله.

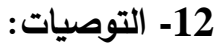

1. تشفير النص المراد إرساله قبل إخفائه بأحدث الطرق ومن أشهرها T-code.

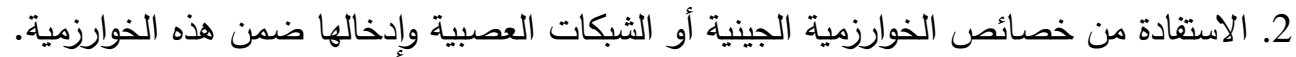




\section{المصادر}

الحمامي، علاء حسين،العاني، سعد عبد العزيز، (2007)، تكنولوجيا أمنية المعلومات وأنظمة الحماية.

[2] Gonzalez, Rafael C.\&woods, Richardv E., 2002,"digital image processing", pearson Education. ISBN 81-7808-629-8.

[3] Babita A. Manpreet K., Manav R. 2009, " High Capacity Filter Based Steganography", International Journal of Recent Trends in Engineering ,vol. 1, No. 1, may 2009.

[4] Khalil I. and shaimaa M. ,2010," Skin classification based on co-occurance matrix" , Raf. J. of comp. \& math s., vol. 7, no. 3, third scxienfific conference information technology.

[5] Ibrahim A., Zabian A.,2009 ,"Algorithm for Text Hiding in Digital Image for information Security", IJCSNS International Journal of computer Scince and Network Security, VOL. 9 NO.6, june 2009.

[6] Namita T. \& Dr. Madhu S., 2010, " Evaluation of variious LSB based of image steganography on GIF file format ", international journal of computer applications (0975-8887) volum 6-no.2, septembar.

[7] Ismat M.,2007, " Digital Alimage processing in remote sensing", final research report no 6/427.

[8] The mathworks, Inc, 2009.

Email: webmaster@mathworks.com 